\title{
Calibration of Parameters for Radio-Frequency Ablation Simulation
}

\author{
N. Kosturski, S. Margenov, and Y. Vutov \\ Institute of Information and Communication Technologies, \\ Bulgarian Academy of Sciences
}

\begin{abstract}
We consider the simulation of the thermal and electrical processes, involved in the radio-frequency (RF) ablation procedure. RF ablation is a low invasive technique for the treatment of hepatic tumors, utilizing AC current to destroy the tumor cells by heating. The procedure consists of inserting an RF probe in the patients liver and attaching a ground pad to the skin. After that the AC current is initiated and maintained for a prescribed duration.

We have conducted experiments with a pork liver and an RF ablation apparatus capable of measuring and recording some of the procedure parameters. Those include the applied power, the effective electrical impedance, and the temperature around the tip of the probe. A history of the values at each second of the test is obtained at the end.

Our aim is to adjust the material properties and other model parameters for the simulation to fit the experimentally obtained results. The electrical conductivity of the tissue can be deduced from the measured power and impedance. After that, we need to determine suitable heat conductivity and capacity coefficients. This is achieved via temperature curves comparison.
\end{abstract}

\section{Introduction}

$\mathrm{RF}$ ablation is an alternative, low invasive technique for the treatment of hepatic tumors, utilizing $\mathrm{AC}$ current to destroy the tumor cells by heating $([7,8])$. The destruction of the cells occurs at temperatures of $45^{\circ} \mathrm{C}-50^{\circ} \mathrm{C}$. The procedure is relatively safe, as it does not require open surgery.

The considered RF probe consists of a stainless steel needle, insulated with polyurethane. The RF ablation procedure starts by placing the RF probe inside the tumor. The surgeon performs this under computed tomography $(\mathrm{CT})$ or ultrasound guidance. Once the probe is in place, RF current is initiated. The surface area of the uninsulated part of the needle conducts RF current.

The human liver has a complex structure, with varying thermal and electrical properties - there are three types of blood vessels with different sizes and flow velocities. Here, we consider a simplified test problem, where the liver consists of one homogeneous hepatic tissue. 

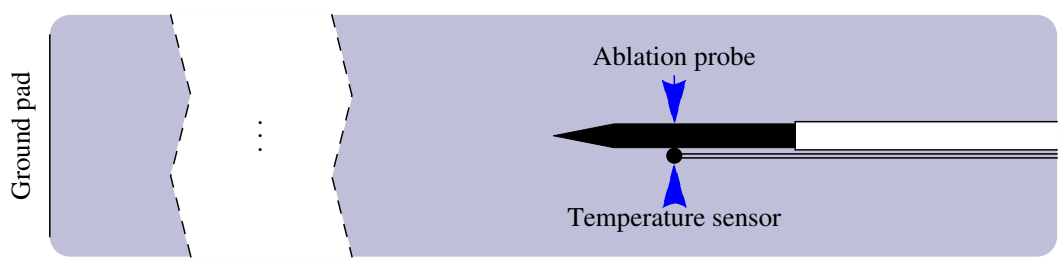

Fig. 1. Experimental Procedure Setup

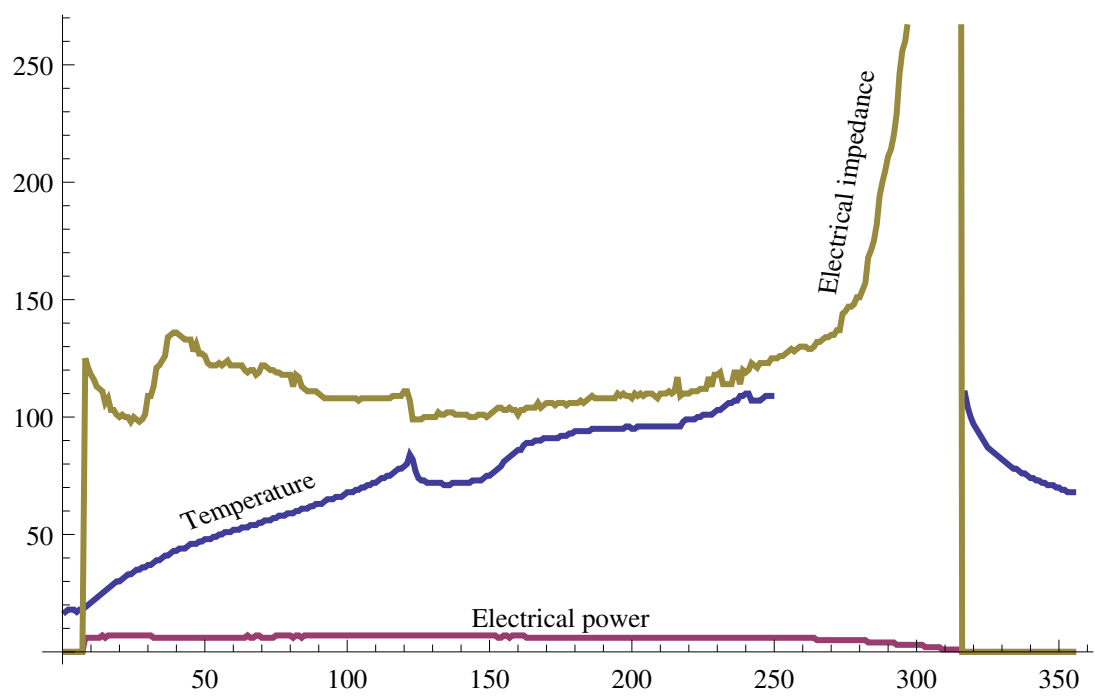

Fig. 2. Measurement Data Obtained during the Experiment

\section{Physical Experiment}

We have conducted experiments with a pork liver and an RF ablation apparatus capable of measuring and recording some of the procedure parameters. The setup is illustrated on Figure 1.

The pork liver was placed inside a metal plate, to which the ground pad was applied. The RF ablation probe was inserted in the liver. After that a temperature sensor was inserted in the hole, created by the probe, parallel to it, and the RF current was initiated. The apparatus measured the applied power, the effective electrical impedance, and the temperature. A history of the values at each second of the test can be seen on Figure 2. Due to limitations of the used temperature sensor, only temperatures below $110^{\circ} \mathrm{C}$ could be measured.

As can be seen, with the increase of the tissue temperature to a certain level, a steep increase of the electrical impedance is observed. This is attributed to tissue charring and vapor formation, which forms an isolating layer around the probe. As a result the electrical power quickly drops to zero and the ablation process 
stops. In this paper, we are concentrating on the ablation process before the impedance jump occurs, in order to check how well the simulation approximates the temperature field. Therefore we are considering the measurement data from the moment electrical current was initiated up to the moment the temperature at the sensor passed $110^{\circ} \mathrm{C}$.

\section{Radio-Frequency Tumor Ablation Model}

Let us turn our attention to the considered numerical simulation. The RF ablation procedure destroys the unwanted tissue by heating, arising when the energy dissipated by the electric current flowing through a conductor is converted to heat. A simplified bio-heat time-dependent partial differential equation $[7,8]$

$$
s \frac{\partial T}{\partial t}=\nabla \cdot k \nabla T+J \cdot E
$$

is used to model the heating process during the RF ablation. The simplification is due to the fact there is no blood perfusion and no metabolic heat production, as the experiment was performed on a dead pork liver. The term $J \cdot E$ in (1) represents the thermal energy arising from the current flow.

The following initial and boundary conditions are applied

$$
\begin{aligned}
& T=T_{0} \text { when } t=0 \text { at } \Omega, \\
& T=T_{0} \text { when } t \geq 0 \text { at } \partial \Omega .
\end{aligned}
$$

The following notations are used in (1) and (2): $\Omega$ is the entire domain of the model, $\partial \Omega$ - the boundary of the domain, $s$ - the volumetric heat capacity $\left(\mathrm{J} / \mathrm{m}^{3} \mathrm{~K}\right), k$ - the thermal conductivity $(\mathrm{W} / \mathrm{m} \mathrm{K}), J$ - the current density $(\mathrm{A} / \mathrm{m}), E$ - the electric field intensity $(\mathrm{V} / \mathrm{m})$, and $T_{0}$ - body (or in this case room) temperature $\left({ }^{\circ} \mathrm{C}\right)$.

The bio-heat problem is solved in two steps. The first step is finding the potential distribution $V$ of the current flow. With the considered RF probe design, the current is flowing from the conducting electrodes to a dispersive electrode on the patient's body. The electrical flow is modeled by the Laplace equation

$$
\nabla \cdot \sigma \nabla V=0
$$

with boundary conditions

$$
\begin{aligned}
& V=0 \text { at } \partial \Omega_{g r}, \\
& V=V_{0} \text { at } \partial \Omega_{e l},
\end{aligned}
$$

where $V$ is the potential distribution in $\Omega, \sigma$ - the electric conductivity ( $\mathrm{S} / \mathrm{m}$ ), $V_{0}$ - the applied RF voltage, $\partial \Omega_{g r}$ - the part of the boundary connected to the ground pad, and $\partial \Omega_{e l}$ - the surface of the conducting part of the RF probe.

After determining the potential distribution, the electric field intensity can be computed from

$$
E=-\nabla V
$$


and the current density from

$$
J=\sigma E .
$$

The second step is to solve the heat transfer equation (1) using the heat source $J \cdot E$ obtained in the first step.

For the numerical solution of both of the above discussed steps of the simulation the Finite Element Method (FEM) in space is used ([2]). Linear conforming elements are chosen in this study. The domain is represented by a voxel image with a resolution of $256 \times 256 \times 256$. To apply the linear FEM discretization to the voxel domain, each voxel is split into six tetrahedra. To solve the bio-heat equation, after the space discretization, the time derivative is discretized via finite differences and the backward Euler scheme is used ([4]).

Let us denote with $K^{*}$ the stiffness matrix coming from the FEM discretization of the Laplace equation (3). It can be written in the form

$$
K^{*}=\left[\int_{\Omega} \sigma \nabla \Phi_{i} \cdot \nabla \Phi_{j} d \mathbf{x}\right]_{i, j=1}^{N},
$$

where $\left\{\Phi_{i}\right\}_{i=1}^{N}$ are the FEM basis functions.

The system of linear algebraic equations

$$
K^{*} X=0
$$

is to be solved to find the nodal values $X$ of the potential distribution.

The electric field intensity and the current density are than expressed by the partial derivatives of the potential distribution in each finite element. This way, the nodal values $F$ for the thermal energy $E \cdot J$ arising from the current flow are obtained.

Let us now turn our attention to the discrete formulation of the bio-heat equation. Let us denote with $K$ and $M$ the stiffness and mass matrices from the finite element discretization of (1). They can be written as

$$
\begin{gathered}
K=\left[\int_{\Omega} k \nabla \Phi_{i} \cdot \nabla \Phi_{j} d \mathbf{x}\right]_{i, j=1}^{N}, \\
M=\left[\int_{\Omega} s \Phi_{i} \Phi_{j} d \mathbf{x}\right]_{i, j=1}^{N} .
\end{gathered}
$$

Then, the parabolic equation (1) can be written in matrix form as:

$$
M \frac{\partial T}{\partial t}+K T=F .
$$

If we denote with $\tau$ the time-step, with $T^{n+1}$ the solution at the current time level, and with $T^{n}$ the solution at the previous time level and approximate the time derivative in (5) we obtain the following system of linear algebraic equations for the nodal values of $T^{n+1}$

$$
(M+\tau K) T^{n+1}=M T^{n}+\tau F .
$$


The matrices of the linear systems (4) and (6) are ill-conditioned and large. Since they are symmetric and positive definite, we use the PCG [1] method, which is the most efficient solution method in this case.

A parallel AMG implementation - BoomerAMG [5,9] is used to precondition the linear systems. The matrix $A=M+\tau K$ from (6) is assembled only once on the first time step and not varied after that. The corresponding AMG preconditioner is also constructed only on the first time step. Additional details concerning the parallelization approach can be found in our paper [6].

\section{Model Parameters Calibration}

Our aim is to identify (tune) the liver tissue parameters, such that the simulation results better fit the results from the physical experiment. We start with the material properties in Table 1, taken from the literature [7].

Before we can calibrate the thermal properties $s_{l}$ and $k_{l}$ of the liver, we need to calibrate it's electrical conductivity $\sigma_{l}$ in accordance with the experimental data. Since the electrical field in the model is static, we first computed the average values for the electrical power and impedance $-P=6.653 \mathrm{~W}$ and $R=111.8 \Omega$ respectively.

In order to match the electrical power $P$, we need to determine the potential $V_{0}$ for the second boundary condition of (3) that will yield the desired value. To do this, the Laplace equation is initially solved with a boundary condition $V=1 \mathrm{~V}$ at $\partial \Omega_{e l}$. Then, $E^{*}$ and $J^{*}$ are obtained from the solution and the corresponding electrical power $P^{*}$ can be computed as

$$
P^{*}=\int_{\Omega} E^{*} \cdot J^{*} d \mathbf{x}
$$

Since the solution and all the components of $E$ and $J$ are proportional to the value of $V_{0}$ we can scale the obtained solution, instead of recomputing it, in the following way

$$
V_{0}=\lambda, \quad E=\lambda E^{*}, \quad J=\lambda J^{*}, \quad \text { where } \quad \lambda=\sqrt{P / P^{*}} .
$$

Now we compute the effective electrical impedance $R^{*}=V_{0}^{2} / P$ and then by setting $\sigma_{l}=0.333 R^{*} / R \approx 0.2494$ and repeating the procedure we obtain a good match of both $P$ and $R$ with a potential $V_{0} \approx 27.28 \mathrm{~V}$.

Table 1. Thermal and Electrical Properties of the Materials

\begin{tabular}{lrrr}
\hline Material & $s\left(\mathrm{~J} / \mathrm{m}^{3} \mathrm{~K}\right)$ & $k(\mathrm{~W} / \mathrm{m} \mathrm{K})$ & $\sigma(\mathrm{S} / \mathrm{m})$ \\
\hline \hline Stainless steel & $2.838 \times 10^{6}$ & 71 & $4 \times 10^{8}$ \\
Liver & $3.816 \times 10^{6}$ & 0.512 & 0.333 \\
Polyurethane & 73150 & 0.026 & $10^{-5}$ \\
\hline
\end{tabular}


After obtaining an electrical field matching the experimental data, we are now ready to calibrate the thermal properties of the tissue. In order to compare the numerical results to the measurement, we run the simulation with a time step of $1 \mathrm{~s}$ and consider the results in a single point that is selected on the boundary between the probe and the tissue around the middle of the uninsulated part of the needle. Let us denote the physically measured temperature on the $i$-th second of the procedure with $T_{i}^{m}$ and the temperature from the simulation with $T_{i}^{s}\left(k_{l}, s_{l}\right)$ respectively. Note that we treat the simulated temperature as a function of the thermal properties of the tissue. Now we can formulate the calibration problem as a least-squares optimization problem

$$
\min \sum_{i}\left(T_{i}^{s}\left(k_{l}, s_{l}\right)-T_{i}^{m}\right)^{2}
$$

Since in the physical experiment the temperature sensor was not firmly attached to the ablation probe, we can attribute drops in the measured temperature to a displacement of the sensor with respect to the probe. Because of this, and also because the selected point normally has the highest temperature we add the following constraints

$$
T_{i}^{s}\left(k_{l}, s_{l}\right) \geq T_{i}^{m}, \quad \forall i
$$

In order to enforce the constraints we use a penalty method, which consists of solving a series of unconstrained minimization problems

$$
\min \Psi^{j}\left(k_{l}, s_{l}\right), \text { for } j=1,2, \ldots
$$

where

$$
\Psi^{j}\left(k_{l}, s_{l}\right)=\sum_{i}\left(T_{i}^{s}\left(k_{l}, s_{l}\right)-T_{i}^{m}\right)^{2}+\theta_{j} \sum_{i} \min \left(0, T_{i}^{s}\left(k_{l}, s_{l}\right)-T_{i}^{m}\right)^{2},
$$

until the minimum stops increasing. The penalty coefficient on the $j$-th iteration is selected as $\left\{\theta_{j}\right\}_{j=1}^{\infty}=\{0,1,10,100, \ldots\}$. Each minimization result is used as an initial guess for the next minimization problem. We use the coefficients from the literature as an initial guess for the first unconstrained minimization. The iterations of the penalty method are illustrated on Figure 3.

For the solution of each unconstrained minimization problem, we selected the principal axis method [3]. It is a derivative-free algorithm, where an approximate model is built up using only values from function evaluations. This algorithm consists of a series of linear searches, with directions, chosen in a way that ensures they are well aligned to the principal directions of a local quadratic model.

In our case each function evaluation meant running an RF ablation simulation with the corresponding material properties and evaluating $\Psi^{j}\left(k_{l}, s_{l}\right)$ from (7) with the resulting temperature samples. For the initial and boundary conditions (2) the value $T_{0}=18^{\circ} \mathrm{C}$ from the measurement before starting the $\mathrm{RF}$ procedure is used.

Each unconstraned minimization required around 150 simulation runs. The constrained minimization had 10 steps. The implementation was run on 512 


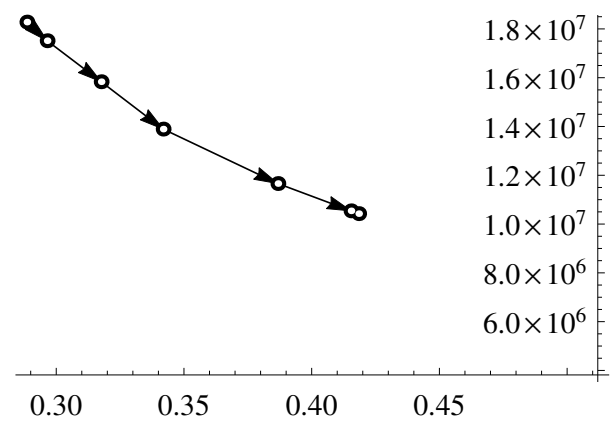

Fig. 3. Penalty Method Iterations

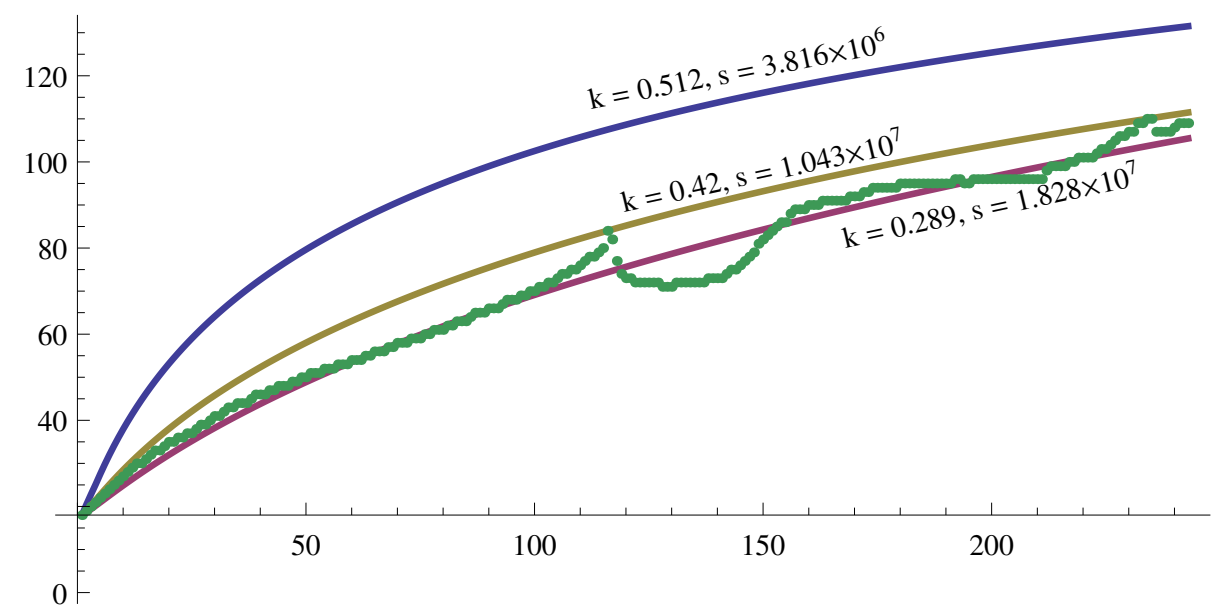

Fig. 4. Simulation Results with Different Coefficients

cores of the IBM Blue Gene/P computer and the maximum job duration of one week was not enough to complete the calibration. However, it was easy to restart the calibration procedure from where it finished and one restart was enough.

The simulation results with the literature coefficients, the unconstrained fit, and the constrained fit can all be seen on Figure 4 along with the corresponding material properties.

\section{Concluding Remarks}

We have described a feasible, albeit time consuming, procedure for calibration of model parameters. No specific assumptions are made, therefore we think the procedure can be applied to any parameters fitting different measurements. In theory, we can calibrate more than two parameters at the same time with the developed implementation, although, this was not tested in practice and the 
performance might be prohibitive. The developed implementation should be very useful as we further complicate our model.

Our next steps would be fitting the simulation to measurements, taken from real patients in clinical trials and also creating a model which includes the experimentally observed impedance increase.

\section{Acknowledglements}

This work is partially supported by the EU Operational Programme "Competitiveness" grant BG161PO003-1.1.06-0004-C0001 and Bulgarian NSF Grant DCVP 02/1. The results in this work are obtained on an IBM BlueGene/P computer located in Sofia, part of the PRACE Research Infrastructure.

We would like to thank Theodor Popov and the engineering team at AMET Ltd. for providing the RF ablation equipment as well as conducting the experiments with us.

\section{References}

1. O. Axelsson, Iterative Solution Methods, Cambridge University Press, 1996.

2. S. Brenner, L. Scott, The mathematical theory of finite element methods, Texts in applied mathematics, 15, Springer-Verlag, 1994.

3. R. Brent, Algorithms for Minimization without Derivatives, Dover, 2002

4. E. Hairer, S.P. Norsett, G. Wanner Solving ordinary differential equations I, II, Springer Series in Comp. Math., 2000, 2002

5. V.E. Henson, U.M. Yang, BoomerAMG: A parallel algebraic multigrid solver and preconditioner, Applied Numerical Mathematics 41 (1), Elsevier, 2002, 155-177.

6. N. Kosturski, S. Margenov, Y. Vutov, Improving the Efficiency of Parallel FEM Simulations on Voxel Domains, Proceedings of LSSC'11, LNCS 7116, pp. 574-581. in Computer Science.

7. S. Tungjitkusolmun, S.T. Staelin, D. Haemmerich, J.Z. Tsai, H. Cao, J.G. Webster, F.T. Lee, D.M. Mahvi, V.R. Vorperian, Three-dimensional finite-element analyses for radio-frequency hepatic tumor ablation, IEEE transactions on biomedical engineering 49 (1), 2002, 3-9.

8. S. Tungjitkusolmun, E.J. Woo, H. Cao, J.Z. Tsai, V.R. Vorperian, J.G. Webster, Thermal-electrical finite element modelling for radio frequency cardiac ablation: Effects of changes in myocardial properties, Medical and Biological Engineering and Computing 38 (5), 2000. 562-568.

9. Lawrence Livermore National Laboratory, Scalable Linear Solvers Project, http: //www.llnl.gov/CASC/linear_solvers/. 